\title{
Research on the development of the centrifugal spinning
}

\author{
Zhang Zhiming , Sun Jun \\ School of Electrical \& Mechanical Engineering, Wuhan Textile University, 430073 Wuhan City, China
}

\begin{abstract}
Centrifugal spinning is a new and efficient method to produce nanofibers quickly. It makes use of the centrifugal force instead of high voltage to produce the nanofibers. The centrifugal spinning has many advantages such as no high voltage, high yield, simple structure, no pollution and can be applied to high polymer material, ceramic and metal material. In order to have more understand about this novel nanofibers formation method, this paper introduces the method of centrifugal spinning and the effect of rotation speed, the properties of material such as viscosity and solvent evaporation, collector distance which have an impact on nanofibers morphology and diameter were also analyzed.
\end{abstract}

\section{Introduction}

Nanofibers are widely used in a lot of fields such as textile industry, medicine, energy and polymer transistors and have attracted much attention because of the high surface area to volume and superior mechanical performance[1-2]. Besides, nanofibers have another character that they have shown great mechanical and thermal properties[3-5]. There are a great deal of ways to produce nanofibers, such as stretching method and electrospinning. Electrospinning is a traditional method to generate nanofibers due to the simple operation and the relatively high production efficiency. Electrospinning is a method in which the solution of the material is forced though a spinneret which is charged at high voltage[6]. Electrostatic force makes the material stretched, when the surface tension is overcome, a jet is ejected from the spinneret, then the jet undergoes a spiral trajectory and finally the jet arrives the collector. Although there are many virtues to the electrospinning process, the low fiber production rates, high voltage $(5 \mathrm{kV} \sim 60 \mathrm{kV})$, solvent limitations and so on make it unable to large scale commercial use[7-10]. In recent years, some progresses have been made to increase the production rate of electrospinning. For instance, modied single-needle, multi-needle, needleless systems have been improved to increase nanofiber production rate. But from some previous research, applying the centrifugal force may result in a great increase in the production rate of nanofibers. Some researchers started to use centrifugal forces from a rotating disk to fabricate nano-scale fibers.

So a new way of nanofibers production which is called centrifugal spinning came up, it has overcome many weaknesses of the electrospinning process and has proved to be a better alternative way for nanofibers formation[11]. Unlike the process of electrospinning, the centrifugal spinning utilizes centrifugal force to produce nanofibers. The principle of the centrifugal spinning is shown in Fig.1.

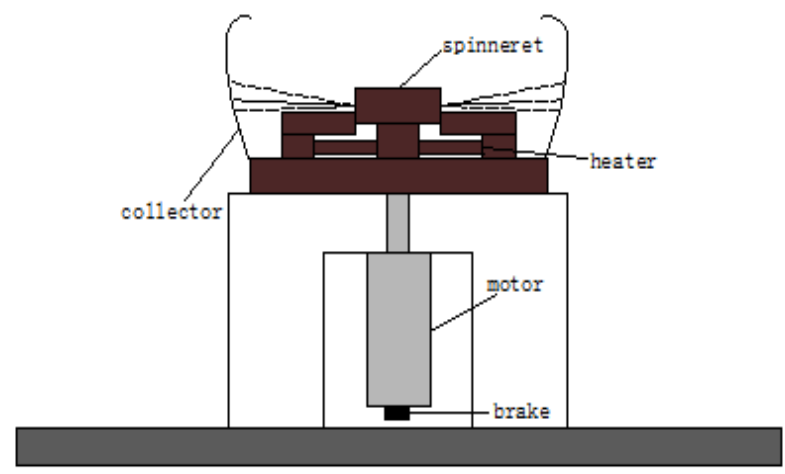

Figure 1. The illustration of the centrifugal spinning

\section{The method of centrifugal spinning}

The equipment of the centrifugal spinning consists of a spinneret containing material which is in solution form such as polymer solutions, then the motor drives the spinneret to spin about its axis of symmetry[12]. When the angular speed is larger than the limitation which is determined by the balance between surface tension and centrifugal force, the material is then expelled out as a jet from a small orifice of the spinneret[13]. If the forces exerted on the jet are too high, the surface tension of the polymer solution will cause the jet to break up and produce beads. This may occur in certain conditions where the angular velocity of the spinneret is low because the centrifugal forces is low and the viscosity of the polymer solution is not too high. As the solvent 
evaporates, the material goes along a curved way. At this moment, the centrifugal force drives the material. Then the material continues to move and finally it reaches the wall of the collector. After the jet expelled out, it forced by the air resistance, so the jet follows a curved trajectory. Air resistance has significant effects on the movement of the liquid jet. An effect of air resistance is the deflection of the jet and causes the jet to progressively bend up. Another effect of air resistance is that it will enhance the surface instabilities. Exposing the jet to the high velocity airflow causes the jet to lose its solvent rapidly and as a consequence, the extension of the jet becomes more difficult. It will also result in thicker nanofibers as the other effect of air resistance. Finally, air resistance could lead to fabricate beaded nanofibers[14]. But it is also said that if both the spinneret and the collector rotate at the same time and direction, air stream will rotates as a rigid body. Therefore, the effects which caused by the air resistance on the jet are negligible. So this method is variable and can be divided into three stages.

\subsection{The first stage}

The first stage of the centrifugal spinning is injecting the material into the spinneret. As mentioned above, there is a motor which drives the spinneret rotating. When the material rotates with the spinneret, the material is forced by many forces, such as friction, gravity, surface tension and centrifugal force, but the surface tension and the centrifugal force take the leading part, others forces are negligible. This stage is shown in Fig.2. As the spinneret rotates, a small solution droplet is seen to originate from the orifice due to the centrifugal forces experienced on the solution within the spinneret. Once the solution droplet reached the lower edge of the orifice, the second process started[15].

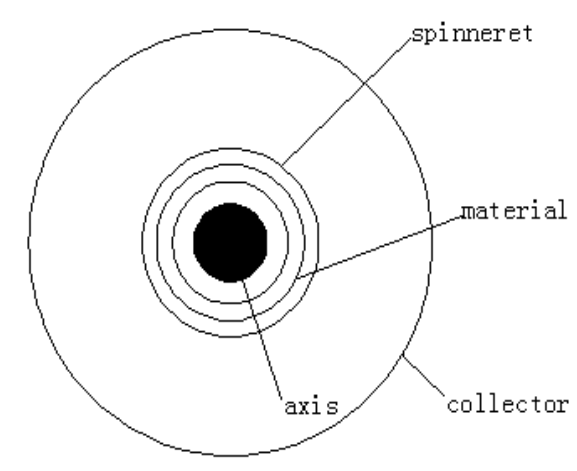

Figure 2. The first stage of the centrifugal spinning

\subsection{The second stage}

The second stage of the centrifugal spinning is that the material is expelled out from the orifice when the centrifugal force is larger than the surface tension[16-17]. Because of the centrifugal force, the polymer solution sustained by its surface tension is explled outward through the orifice.
The centrifugal force of the material can be expressed as equation(1).

$$
F=m r \omega^{2}
$$

Where $m$ is the mass of the material, $r$ is the radius of the spinneret, $\omega$ is the speed of the spinneret.

This equation shows that there is a critical angular velocity exists that produces a jet.

After this stage, the material will go into the third stage.

\subsection{The third stage}

The third stage of the centrifugal spinning is the stage that the nanofibers form. After the material expelled out from the spinneret, it will go along a curve way with the solvent is evaporating, at the same time, the inertia force will make the material stretch. So the diameter of the material will become smaller and smaller, and finally when it reaches the collector, it forms the nanofibers. The third stage is shown in Fig.3.

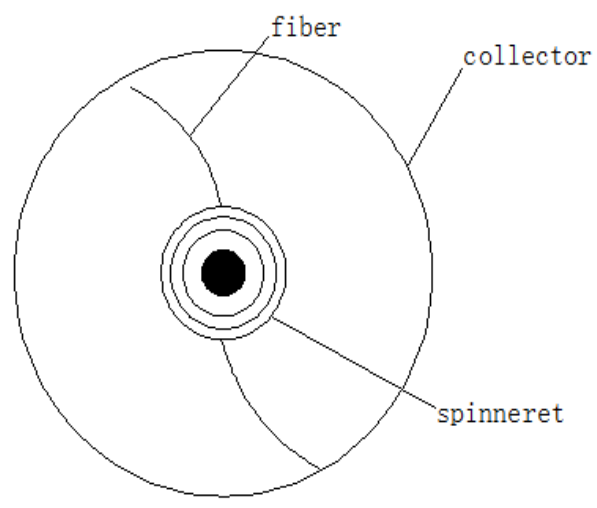

Figure 3. The third stage of the centrifugal spinning

This great new method of nanofibers production relies on the centrifugal force, so it can be used to other new materials that are impossible with the electrospinning. The production efficiency of the centrifugal spinning is much higher than electrospinning. By summarizing, fiber formation requires a critical angular velocity because only if it is reached, a jet can be produced. These conditions have been established in the fiber formation part. Afterwards, another angular velocity must be established at which the fiber reaches what will from now on be described as the trajectory of fiber. During trajectory, the fiber reaches a stable position during which the fiber continues to be stretched without a significant change in its distance to the collector. It is during this period that the fiber diameter experiences the most reduction. It is also during this time that the fiber experiences vibration in the form. After several spinneret rotations, the fiber diameter is reduced considerably and begins to once again expand outwards to eventually reach the collector. Finally, a collector is used to gather the produced fibers. 


\section{Influence of material parameters to nanofibers morphology}

Understanding the three stages of this method for nanofibers formation, it will provide the proper choose of material properties such as viscosity and surface tension, operating parameters such as angular velocity, temperature, machine devices such as spinnerets and collectors. When using centrifugal spinning to produce nanofibers, the material must be in solution or melt form. And these characters of the solution are important in determining the final nanofibers diameter and morphology[18]. There are many parameters that affect the quality of nanofibers. At the process of nanofibers production, two significant material parameters which are the surface tension and viscosity should be considered. Besides, there are some others parameters that should also be considered, such as the speed of the spinneret, the distance of the collector and the diameter of the orifice[19-20]. The other parameter such as initial polymer concentration, solution density, perturbation frequency and solvent vapour pressure has been found to be moderate, so these parameters can be neglected.

\subsection{Surface tension}

In the centrifugal spinning, the surface tension of the material is a key parameter in nanofibers production. The centrifugal force and the surface tension together affect the formation of nanofibers[21-22]. At the second stage of the centrifugal spinning, the surface tension must be overcome by centrifugal force so the solution of material can form the Taylor cone at the orifice. The surface tension will make surface energy of the solution less and this will lead to the formation of droplets rather than the nanofibers, but the centrifugal force can connect the droplets together and make sure that the nanofibers will not break, so the centrifugal force is the key in the process of nanofibers formation. When other parameters are proper, decreasing the surface tension of the material will help to form the nanofibers.

\subsection{Viscosity of solution}

Another parameter that must be controlled for good fiber formation is the viscosity of the polymer melt or solution. Due to the large viscosity, the forces being used to draw the fiber may not be strong enough to create a jet. On the other hand, the viscosity is low, the jet may break up and produce beads instead of fibers. For the polymer solution, if the viscosity of solution is too large, the gravitation between molecules will be large and it will make the molecules tangle together, the external force cannot make the solution expel out from the spinneret and the nanofibers cannot be produced too[23]. If the viscosity of solution is too small, the droplet will form or the material will break in the third stage of the centrifugal spinning. The number of the viscosity of solution is related to the temperature and the properties of solution. Besides, the viscosity of solution will affect the velocity of the solution and the morphology of the nanofibers. When the velocity of the solution increases, the diameter of nanofibers will decrease.

\subsection{The speed of the spinneret}

The speed of the spinneret is also an important parameter that will determine the nanofibers production, because the solution is forced by the surface tension and centrifugal force, when the speed of the spinneret is small, the centrifugal force can not overcome the surface tension, the solution will be stuck in the spinneret. So the speed of the spinneret should be proper. When the speed is proper, the quality and morphology of the nanofibers are good[24]. So the centrifugal force plays a leading role in the process of nanofibers formation, when the speed of the spinneret increase in a right range, the diameter of the nanofibers will decrease. Some experiments show that the $14000 \mathrm{rpm}$ is the best speed to produce nanofibers. It is said that when the speed of the spinneret is increased and the trajectory is expanded, the fiber diameters are reduced, and the fibers produced become more uniform in size.

\subsection{The distance of the collector}

The distance of the collector means the distance between the spinneret and the collector. When only the distance of the collector is changed, the diameter of the nanofibers does not change much, but there are some changes in the morphology of the nanofibers: the nanofibers are more twisted and broken[25-26]. When the speed of the spinneret increases, the trajectory of the material will expand, so the distance of the collector should also increase. When the viscosity of the solution increases, the speed of the nanofibers will decrease, so the distance of the collector should decrease. But if the distance of the collector is too short, the fiber will not be allowed to stretch enough and then the diameter of the produced fiber will be large. How to decide the most appropriate distance is only depended on how long it will take for the solvent to evaporate, the distance required to allow the fiber to begin to be expelled outwards and the model of collection method used.

\subsection{The evaporation rate}

The evaporation rate of the polymer solution jet means much in centrifugal spinning. As the jet is expelled out from the spinneret and went toward the collector, the fiber would go on a rapid evaporation which also decreased the diameter of the fiber. If the evaporation rate of the solution is too low, then the fibers may be converted to a thin film as the layers build up on the collector. If the evaporation rate is too high, then the third process of the fiber formation is disturbed and finally it will produce fibers with large diameters[27]. This is dependent of a lot of factors such as the rate of heat supply, boiling point, surface tension of liquid, vapor pressure, interaction between solvent and solute molecules, and air movement on the solution surface. 


\subsection{The diameter of the orifice}

The diameter of the orifice directly determines the flow of the solution and the initial diameter of the fiber. When the orifice is too large, it will result in droplet forming[28]. When the diameter of the orifice is adequately small, it is easier to produce the nanofibers. Some researches indicated that decreasing the orifice diameter decreases the final diameter of the collected fibers. Studying the effects of the various parameters in centrifugal spinning, it was found that the two most influential parameters are the angular velocity of the spinneret and the viscosity of the solution.

\section{Conclusions}

This paper introduces the method of centrifugal spinning and the three stages of it and also briefly describes the advantages of the centrifugal spinning. And in this paper, the centrifugal spinning and the electrospinning are compared. The centrifugal spinning is a more proper way to produce nanofibers, although there are some achievements, this method also has a lot of problems that need to be studied. For example, the parameters that affect the nanofibers production and the morphology of nanofibers are already analyzed, but due to the complexity of these factors, the mathematical model that can describe the relationship between these parameters and the diameter of nanofibers is not established, and there is no effective way to solve the problems that are relation to the effects of solvent evaporation and temperature on fiber diameter and morphology. But with the development of science and technology, there will be more and more researches on these problems and the centrifugal spinning will finally be widely used.

\section{References}

1. R. Nayak, R. Padhye, I. L. Kyratzis, Textile Research Journal,82,129(2012)

2. S.Ramakrishna,K.Fujihara,W.E.Teo,World Scientific Publishing Company,(2006)

3. Y. Ito, H. Hasuda, M. Kamitakahara, Journal Of Bioscience and Bioengineering, 100,43(2005)

4. J. Yoon, Y. S. Jung, J. M. Kim, Advanced Functional Materials, 19,209(2009)
5. S.Ramakrishna, K.Fujihara, V.K.Ganesh, American Scientific Publisher, 7(2006)

6. A. Baji, Y. Mai, S. C. Wong,Composites Science and Technology,70,703(2010)

7. K. Sarkar, C. Gomez, S. Zambrano, Materials Today, $13,11(2010)$

8. Z. Mceachin, K. Lozano, Journal of Applied Polymer Science, $126,473(2012)$

9. T. Senthilram, L. A. Mary, J. R.Venugopal,Materials Today, 14,226(2011)

10. X. W. Zhang, Y. Lu, Polymer Reviews,54,677(2014)

11. E. Kolk, Texas Center for Educational Research,23, 429(2005)

12. Z. M. Zhang, Q. Xu, S. Q. Mei. Polymer Bulletin, 5, 29(2013)

13. P. Mellado, H. A. Mcilwee, Applied Physics Letters, 99,20(2011)

14. J L. Cummings, D P. Howell, Journal of Fluid Mecha nics, 4,361(1999)

15. A. Valipouri, S.A.H. Ravandi, A.R. Pishevar, Fibe rs and Polymers, 14(6),941-949(2013)

16. S. P. Decent, A. C. King, I. M. Wallwork, Journal of Engineering Mathematics, 42,265(2002)

17. I. M. Wallwork, S. P. Decent, A. C. King, Applied M athematical Modelling,33,4283(2002)

18. S. Padron, D. Caruntu, K. Lozano, Asme Internation al Mechanical Engineering Congress \& Exposition, 821(2011)

19. S. A. Theron, A. L. Yarin, E. Zussman, Polymer,46, 2889(2005)

20. D. H. Reneker, A. L. Yarin, H. Fong, Journal of Applied Physics, 87,4531(2000)

21. H. Z. Xu, H. H. Chen, X. G. Li, Technical Textiles, 1 , 25(2016)

22. S.Ramakrishna, T.C.Lim, R.Inai, Mechanics of Advanced Materials \& Structures, 13(1),77-81(2006)

23. T. D. Song, X. H. Li, Y. Liu, China Textile Leader,1, 61(2014)

24. N. E. Zander, Journal Of Applied Polymer Science, 132,2(2015)

25. Y. Lu, Y. Li, S. Zhang, Polymer Journal, 49,3834 (20 13)

26. X. Li, H. Liu, J. Wang, Polymer,53,248(2012)

27. S. Padron, A. Fuentes, D. Caruntu, K. Lozano, Journ al of Applied Physics, 113(2),24318(2013)

28. S. Parden, A. Fuentes, Journal of Applied Physics, 113,1(2013) 